\section{SDF1 $\alpha$-induced chemotaxis of JAK2-V617F-positive cells is dependent on Bruton tyrosine kinase and its downstream targets PI3K/AKT, PLC 11 and RhoA}

JAK2-V617F is present in nearly $95 \%$ of patients with polycythemia vera and $50-60 \%$ of those with essential thrombocythemia or primary myelofibrosis. ${ }^{1}$ In addition to erythrocytosis, thrombocytosis and bone marrow fibrosis (as in polycythemia vera, essential thrombocythemia, and primary myelofibrosis, respectively), the clinical course of patients with myeloproliferative neoplasia (MPN) is characterized by increased risks of thrombosis, splenomegaly and an inflammatory response syndrome. ${ }^{2,3}$ Clinical studies of JAK kinase inhibitors have shown that these agents can produce considerable improvements of splenomegaly, constitutive symptoms, and overall survival in MPN patients. ${ }^{3}$ However, the therapeutic response is often limited and short-lived. In addition, transformation to acute leukemia remains a major problem. It is, therefore, essential to identify novel nodes of constitutive JAK2-V617F signaling and to develop better approaches to the therapy of these neoplasias. ${ }^{4}$ Downstream of JAK2-V617F, NFKB signaling (p65) is constitutively active and regulates expression of CXCL10 in MPN. ${ }^{5}$ In bortezomib-resistant multiple myeloma cells ${ }^{6}$ and in acute myeloid leukemia cells with MLL-AF9 rearrangements, ${ }^{7}$ constitutively active $\mathrm{NF \kappa B}$ drives the expression of Bruton tyrosine kinase (BTK). Of note, it was found that BTK interacted with erythropoietin receptor-JAK2 and was tyrosine phosphorylated in response to treatment with erythropoietin. ${ }^{8}$ These observations led us to investigate whether JAK2-V617F kinase also induces BTK expression (via p65) and activation and to characterize its physiological relevance in JAK2V617F-positive cells.

To investigate whether BTK is activated by JAK2V617F, we used 32D myeloid progenitor cells ectopically expressing human erythropoietin receptor and JAK2wildtype (32D JAK2-WT) or JAK2-V617F (32D JAK2V617F). ${ }^{9}$ Tyrosine phosphorylation of BTK was indeed elevated in whole cell lysates of 32D JAK2-V617F cells compared to the level in 32D JAK2-WT cells (Figure 1A). Consistent with this finding, erythropoietin additionally induced BTK phosphorylation in 32D JAK2-WT cells (Online Supplementary Figure S1A). Of note, BTK was overexpressed in 32D JAK2-V617F cells. In line with our
A

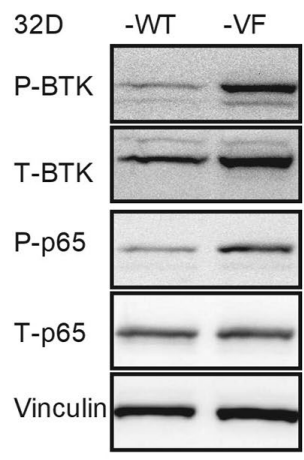

C

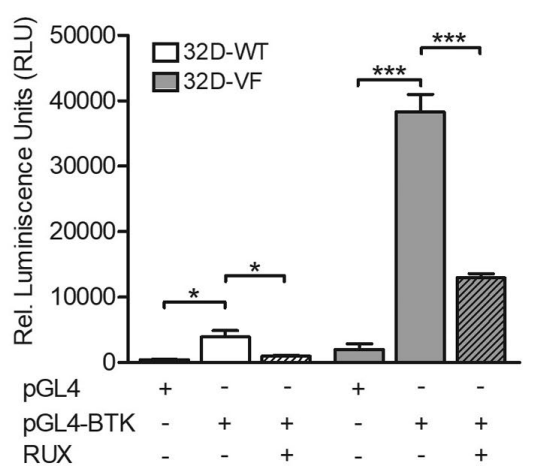

B

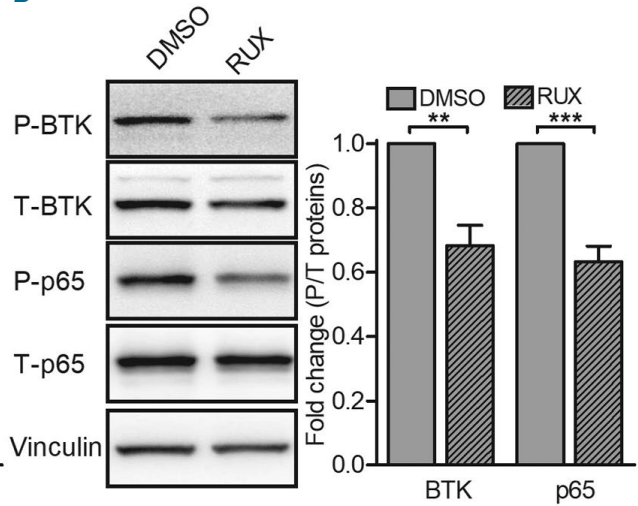

D
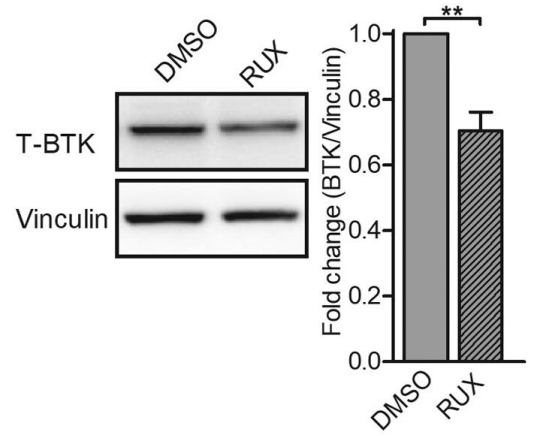

Figure 1. Bruton tyrosine kinase is overexpressed and constitutively active in 32D myeloid progenitor cells ectopically expressing erythropoietin receptor and JAK2-V617F kinase. (A) 32D JAK2-wildtype (WT) and JAK2-V617F (VF) cells were starved of serum for $4 \mathrm{~h}$ and the whole cell lysates were analyzed for expression of phosphorylated (P) Bruton tyrosine kinase (BTK) (pY223) or p65 (pS536) and total (T) proteins. Densitometric analysis of P/T-BTK and -p65 is shown ( $\mathrm{n}=5$ ). (B) 32D VF cells were cultured in the presence of dimethyl sulfoxide (DMSO) or $0.5 \mu \mathrm{M}$ ruxolitinib (RUX) overnight and the whole cell lysates were analyzed for expression of P-BTK or -p65 and T proteins. Densitometric analysis of P/T-BTK and -p65 is shown $(n=4)$. (C) 32D WT and VF cells were electroporated with control (pGL4) or BTK promoter construct (pGL4-BTK) and treated with DMSO or $0.5 \mu \mathrm{M}$ RUX overnight and promoter assays were performed as described previously $^{5}(n=3)$. (D) 32D VF cells were cultured in the presence of DMSO or $0.5 \mu M$ RUX overnight and whole cell lysates were analyzed for expression of BTK. Densitometric analysis of T-BTK relative to vinculin is shown $(n=4)$. Vinculin served as a loading control in all the experiments. Columns represent mean \pm standard error of mean from independent experiments. Statistical significance between different conditions was calculated using the Student $t$-test; $* P<0.05$, $* * P<0.01$ and $* * * P<0.001$. 
previous observations, ${ }^{5}$ 32D JAK2-V617F whole cell lysates demonstrated constitutive activation of p65 (Figure 1A). Emphasizing the contribution of the constitutively active JAK2-V617F kinase, the JAK1/2 inhibitor ruxolitinib inhibited phosphorylation of both BTK and p65 in 32D JAK2-V617F cells (Figure 1B). These findings were confirmed in human cell lines with the JAK2-V617F mutation (Online Supplementary Figure S1B). HEL and SET2 (with the JAK2-V617F mutation) demonstrated strong expression and activation of BTK when compared to OCI AML3 (without the JAK2-V617F mutation). MOLM13 cells (with the FLT3-internal tandem duplication) served as a positive control for BTK expression. The contribution of activated p65 in BTK expression, ${ }^{6,7}$ together with the observations presented in Figure 1A (p65 activation and BTK overexpression), led us to hypothesize a role for activated p65 in BTK expression. Luciferase-based BTK promoter assays were performed by electroporating 32D JAK2-WT and JAK2-V617F cells with an empty construct (pGL4) or test construct (pGL4BTK promoter). ${ }^{6}$ The BTK promoter region in the test construct harbors two p65 binding sites. ${ }^{10}$ In comparison to JAK2-WT cells, 32D JAK2-V617F cells demonstrated elevated luciferase activity, indicating enhanced transcriptional activity of p65 on the BTK promoter (Figure 1C). Confirming a role for JAK2-V617F kinase in p65 activity, ruxolitinib treatment negatively affected BTK promoter activity (Figure 1C) and protein expression (Figure 1D). Thus, downstream of JAK2-V617F signaling, our data suggest a novel role for activated p65 in the regulation of BTK expression.

Growing evidence supports an important contribution of BTK in cell migration via the stromal cell-derived factor 1 alpha (SDF1 $\alpha$ )-CXCR4 axis. ${ }^{11,12}$ BTK mediates activation of phospholipase C gamma (PLC $\gamma) 2$ and regulates SDF1 $\alpha$-induced migration and homing of $B$ cells to the spleen and bone marrow. ${ }^{11}$ We showed earlier that erythropoietin receptor-JAK2-dependent PLC $\gamma 1$ activation is
A

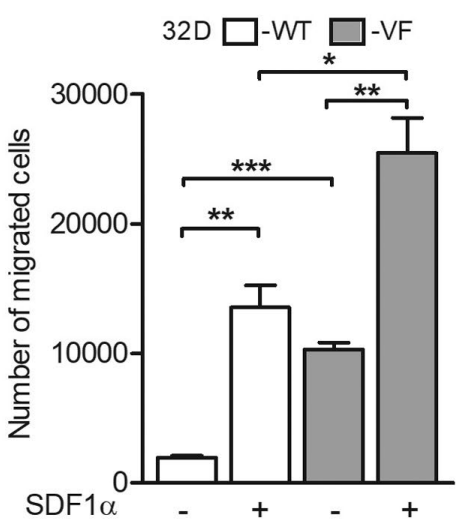

B

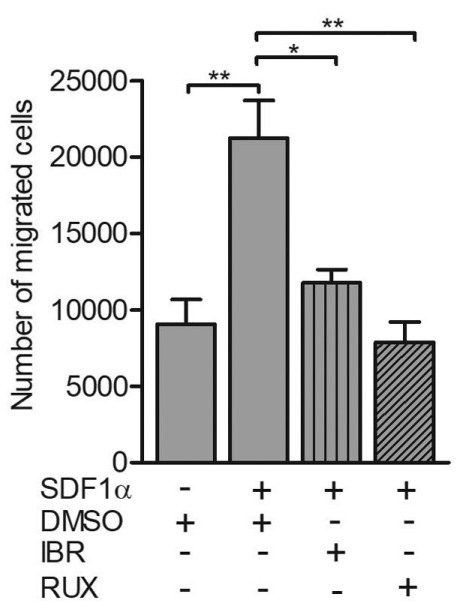

C

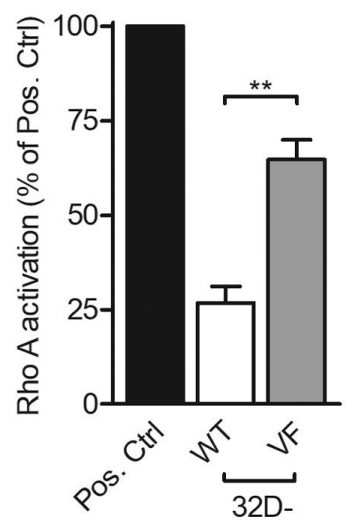

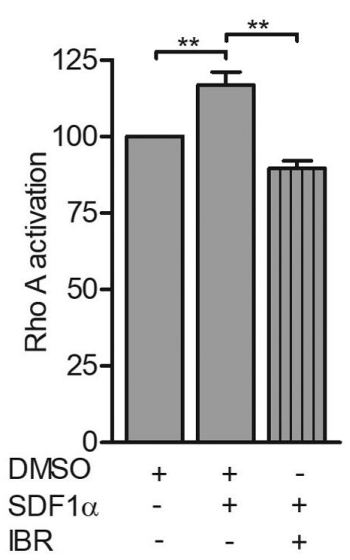

D

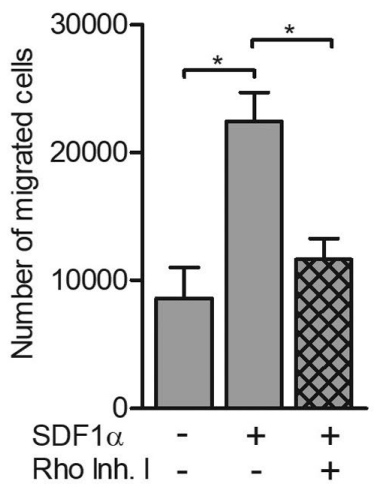

Figure 2. Bruton tyrosine kinase mediates stromal cell-derived factor $1 \alpha$-induced chemotaxis via activation of the small GTPase RhoA. (A, B) 32D JAK2-wildtype (WT) and JAK2-V617F (VF) cells were starved of serum for $2 \mathrm{~h}$ and left alone $(\mathrm{n}=3)$ or treated with dimethyl sulfoxide (DMSO) and inhibitors against Bruton tyrosine kinase (BTK) ( $2 \mu \mathrm{M}$ of ibrutinib: IBR) and JAK2 ( $1 \mu \mathrm{M}$ of ruxolitinib: RUX) as indicated for an additional $2 \mathrm{~h}(\mathrm{n}=4)$ and chemotaxis assays were performed using transwells (pore size: $5 \mu \mathrm{m})$. Starvation medium ( $0.5 \%$ fetal bovine serum) containing stromal cell-derived factor 1 alpha (SDF1 $\alpha$ : $100 \mathrm{ng} / \mathrm{mL})$ served as the chemoattractant. (C) Active RhoA was determined by the G-LISA RhoA activation kit (Cytoskeleton \#BK124) following the manufacturer's instructions. 32D WT and VF cells were starved of serum for $4 \mathrm{~h}$ and left alone (left panel) $(\mathrm{n}=4)$ or treated with DMSO and $1 \mu \mathrm{M}$ IBR as indicated (right panel) ( $\mathrm{n}=4$ ). After $1 \mathrm{~h}$, the cells were stimulated with SDF1 $\alpha(100 \mathrm{ng} / \mathrm{mL})$ for an additional $2 \mathrm{~h}$ and whole cell lysates were analyzed for RhoA activation. (D) 32D VF cells were treated with $2 \mu \mathrm{M}$ of Rho inhibitor (Rho Inh.I) for $4 \mathrm{~h}$ in serum starvation medium as indicated and chemotaxis assays were performed using transwells (pore size: 5 $\mu \mathrm{m})(\mathrm{n}=3)$. Starvation medium ( $0.5 \%$ fetal bovine serum) containing SDF1 $\alpha(100 \mathrm{ng} / \mathrm{mL})$ served as the chemoattractant. Columns represent mean \pm standard error of mean from independent experiments. Statistical significance between different conditions was calculated using the Student $t$-test; $* P<0.05, * \star P<0.01$ and $* * * P<0.001$. 

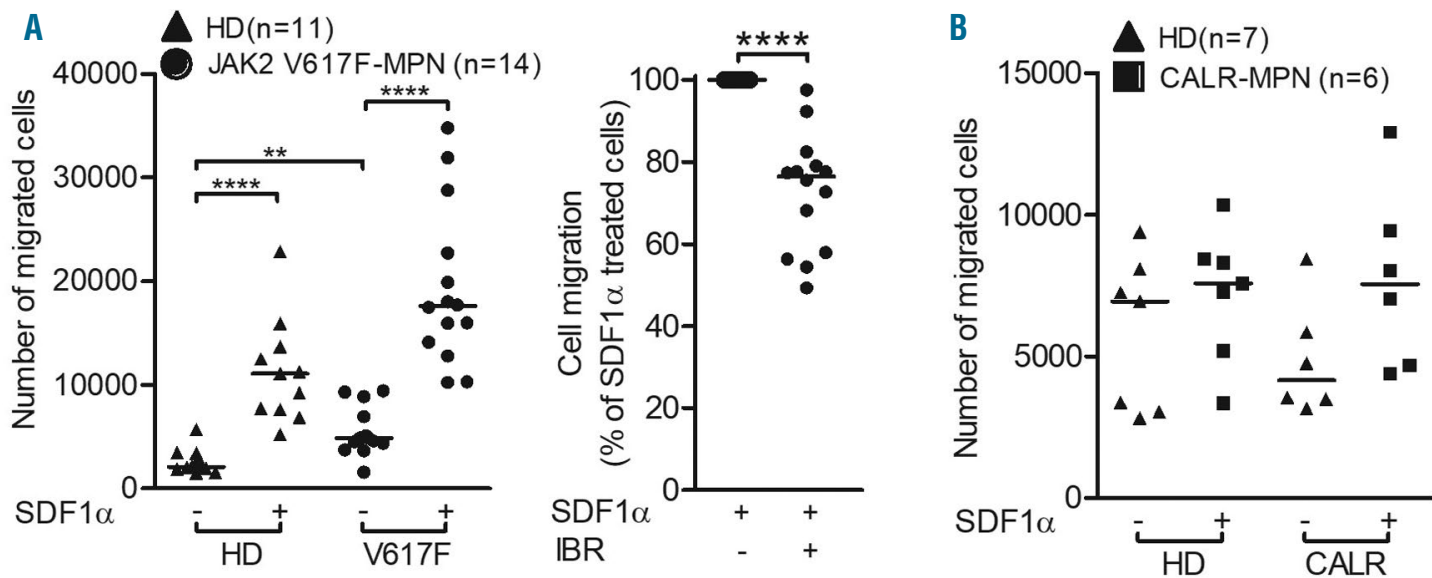

Figure 3. Stromal cell-derived factor 1 alpha-induced chemotaxis of primary granulocytes isolated from peripheral blood of JAK2-V617F-positive patients with myeloproliferative neoplasms could be impeded by a clinically relevant dose of ibrutinib. Granulocytes were isolated (Ficoll-paque density gradient centrifugation- erythrocyte lysis based method) from peripheral blood of healthy donors ( $\mathbf{\Delta}$ : HD), and patients with untreated JAK2-V617F-positive ( $\bullet$ : JAK2 V617F-MPN) (A) or CALR-mutated (ם: CALR-MPN) (B) myeloproliferative neoplasia (MPN). The protocol was approved by the local ethics committee (protocol n. MD115/08), and all patients signed informed consent. The cells were rested for $30 \mathrm{~min}$ in starvation medium $(0.5 \%$ fetal bovine serum) and left alone (A, left panel and $\mathrm{B})$ or treated with dimethylsulfoxide (DMSO) or $0.5 \mu \mathrm{M}$ of ibrutinib (IBR) (A, right panel) for an additional $1 \mathrm{~h}$ and chemotaxis assays were performed (pore size: 3 um). Starvation medium (0.5\% fetal bovine serum) containing stromal cell-derived factor 1 alpha (SDF1 $\alpha$ : $100 \mathrm{ng} / \mathrm{mL})$ served as the chemoattractant. Chemotaxis of IBR-treated cells was normalized to that of DMSO-treated cells. The horizontal lines indicate medians and the statistical significance of differences between control and treated samples was calculated by the Mann-Whitney test; $* P<0.05, * * P<0.01, * * * P<0.001$ and $* * * * P<0.0001$.

essential in erythroid progenitor differentiation. ${ }^{9}$ Recently it was demonstrated that MPL-W515L or cytokine-dependent JAK2 activation cooperates with SDF1 $\alpha$-CXCR4 signaling to enhance chemotactic response via phosphoinotiside 3 kinase (PI3K) signaling. ${ }^{13}$ These observations led us to investigate whether PLC $\gamma 1$ and PI3K signaling are activated downstream of the JAK2-V617F-BTK signaling cascade and whether they contribute to cell migration. 32D JAK2-V617F cells were treated with a BTK inhibitor (ibrutinib) or ruxolitinib and were analyzed for AKT and PLC $\gamma 1$ phosphorylation. Positioning BTK upstream of PI3K-AKT and PLC $\gamma 1$, ibrutinib treatment inhibited AKT and PLC $\gamma 1$ phosphorylation (Online Supplementary Figure S2A,B). Plasma and splenic SDF1 $\alpha$ levels are elevated in patients with polycythemia vera and primary myelofibrosis. ${ }^{14}$ Consequently, we hypothesized that SDF1 $\alpha$-dependent cell migration occurs via BTK and its downstream signaling nodes PI3K-AKT and PLC $\gamma 1$ in JAK2-V617F cells. Basal migration of 32D JAK2-V617F cells was significantly higher than that of 32D JAK2-WT cells (Figure 2A). Supporting this observation, eyrthropoietin-induced JAK2 activation enhanced chemotaxis in 32D JAK2-WT cells (Online Supplementary Figure S3A). Erythropoietin in combination with the chemokine of interest, SDF1 $\alpha$, failed to promote further chemotaxis in 32D JAK2-V617F cells (Online Supplementary Figure S3B). Interestingly, our data also demonstrate a collaboration between JAK2 and SDF1 $\alpha$ signaling in enhancing chemotaxis of both 32D JAK2-WT and JAK2-V617F cells (Figure 2A). Underscoring the contribution of activated BTK, ibrutinib blocked basal cell migration in 32D JAK2-V617F cells (Online Supplementary Figure S3C). Furthermore, ruxolitinib and ibrutinib attenuated SDF1 $\alpha$-induced chemotaxis in these cells (Figure 2B). This observation could be recapitulated in BaF3 JAK2-V617F cells (Online Supplementary Figure S3D). Demonstrating an important role for activated PLC $\gamma 1$ and PI3K-AKT signaling down- stream of JAK2-V617F and activated BTK (Online Supplementary Figure S2A,B), PI3K-AKT and PLC $\gamma 1$ inhibitors (LY294002 and U73122, respectively) suppressed SDF1 $\alpha$-induced chemotaxis. Ibrutinib in combination with LY294002 or U73122 additionally inhibited SDF1 $\alpha$-induced chemotaxis (Online Supplementary Figure $S 3 E$ ). Importantly, ibrutinib treatment failed to affect either basal or SDF1 $\alpha$-induced cell migration in JAK2V617F-negative control cells (primary T-lymphocytes and $32 \mathrm{D}$ parental cells) (Online Supplementary Figure $S 4 A, B$ ). In contrast, ruxolitinib blocked SDF1 $\alpha$-induced cell migration in these cells. Since p65 transcriptionally regulated BTK expression, we investigated whether p65 inhibition could affect cell migration. NFKB inhibitors (BAY11-7082 and IKK inhibitor VII) did indeed block basal and SDF1 $\alpha$-induced cell migration in 32D and BaF3 JAK2-V617F cells (Online Supplementary Figure $S 4 C, D$ ). Since BTK is activated downstream of the JAK2-V617F mutation and ibrutinib has been reported to have a cytotoxic effect, ${ }^{7,15}$ we examined the therapeutic potential of this drug (Online Supplementary Figure S4E). 32D JAK2V617F cells did not respond to ibrutinib even after $48 \mathrm{~h}$, suggesting a cell survival-independent function of BTK activation in these cells.

To exclude potential off-target effects associated with the PLC $\gamma$ inhibitor U73122, we evaluated cell migration in 32D JAK2-V617F cells upon PLC $\gamma 1$ knockdown (Online Supplementary Figure S5A). Both the short hairpin RNA (\#14, \#133) tested successfully knocked down PLC $\gamma 1$ (left panel) and this resulted in suppression of SDF1 $\alpha$-induced chemotactic migration (right panel). Importantly, SDF1 $\alpha$ significantly induced chemotaxis in 32D JAK2-V617F cells but not in cells with PLC $\gamma 1$ knockdown. This observation intrigued us and led us to investigate whether SDF1 $\alpha$ induced chemotaxis via PLC $\gamma 1$ phosphorylation. In support of this possibility, SDF1 $\alpha$ significantly enhanced phosphorylation on PLC $\gamma 1$ (Online Supplementary Figure S5B). Knockdown of PLC $\gamma 1$ in con- 
trol cells (32D and BaF3 parental cells) did not affect basal migration but negatively affected SDF1 $\alpha$-induced cell migration (Online Supplementary Figure S5C,D). The data thus indicate an important role for PLC $\gamma 1$ activation downstream of JAK2-V617F kinase or SDF1 $\alpha$ signaling. Since SDF1 $\alpha$ induced significant chemotaxis in 32D JAK2-WT and JAK2-V617F cells, we were intrigued to evaluate its receptor CXCR4 expression. 32D JAK2-WT and JAK2-V617F cells demonstrated the highest and lowest surface expression of CXCR4, respectively (Online Supplementary Figure $S 6 A$ ). Erythropoietin resulted in a dose-dependent decrease of surface CXCR4 expression in 32D JAK2-WT cells. Hence, JAK2 activation regulates chemotaxis potentially via inducing changes in CXCR4 turnover on the cell surface. Although ibrutinib inhibited cell migration, it failed to affect CXCR4 expression (Online Supplementary Figure S6B). Ibrutinib thus affects cell migration by blocking intracellular signaling in JAK2V617F cells.

We next investigated the signaling molecules influencing SDF1 $\alpha$-controlled migration downstream of BTK. Ras homolog gene family, member A (RhoA) activation is important in the regulation of cytoskeletal dynamics and cell migration in diverse cell types. In Jurkat cells, SDF1 $\alpha$ induced activation of RhoA and RhoC plays a pivotal role in the regulation of chemotaxis. ${ }^{16}$ Furthermore, a role for the BTK family of kinases in the regulation of Rho GTPases has been described. ${ }^{17}$ Quantitative mass spectrometry-based investigations of the granulocyte proteome revealed an underexpression of Rho-GDP dissociation inhibitors (Rho-GDI 1 and 2) in JAK2-V617F-positive $\mathrm{MPN} .^{18}$ Since Rho-GDI negatively regulate Rho GTPases, we hypothesized an increased RhoA activation downstream of JAK2-V617F kinase. RhoA GTPase activation assays, which specifically recognize active GTPbound forms, were performed in 32D JAK2-WT and JAKV617F cells. RhoA activation was indeed elevated in 32D JAK2-V617F cells in comparison to that in 32D JAK2-WT cells (Figure 2C, left panel). Underscoring the contribution of activated JAK2, erythropoietin induced RhoA activation in 32D JAK2-WT cells (Online Supplementary Figure $S 7 A)$. SDF1 $\alpha$ enhanced basal RhoA activation in 32D JAK2-V617F cells and pretreatment of the cells with ibrutinib significantly attenuated SDF1 $\alpha$-induced RhoA activation (Figure $2 \mathrm{C}$, right panel). RhoA activation was validated in granulocytes of MPN patients with JAK2-V617F mutation (Online Supplementary Figure $S 7 B$ ). In response to external stimuli, RhoA regulates cell migration via actin remodeling. ${ }^{19}$ Establishing a role for RhoA activation in cell migration, 32D JAK2-V617F cells demonstrated increased actin polymerization (F-actin) (Online Supplementary Figure S8A). SDF1 $\alpha$ further augmented Factin staining in these cells. Granulocytes from JAK2V617F MPN patients demonstrated higher actin polymerization than those from healthy donors (Online Supplementary Figure $S 8 B$ ). Confirming an essential role for RhoA activation, Rho inhibitor treatment blocked SDF1 $\alpha$-induced cell migration in 32D JAK2-V617F cells (Figure 2D). Collectively our observations highlight RhoA GTPase as one of the essential downstream targets of JAK2-V617F-BTK signaling in regulating cell migration.

Finally, we sought to validate our cell migration results in primary granulocytes isolated from the peripheral blood of healthy donors, JAK2-V617F-positive and CALR-mutated MPN patients. Supporting our cell migration data using 32D and BaF3 JAK2-V617F cells, granulocytes isolated from JAK2-V617F MPN patients demonstrated increased basal migration (2.1-fold higher) as compared to granulocytes from healthy donors (Figure
$3 A$, left panel). SDF1 $\alpha$ treatment further enhanced migration in granulocytes from patients with MPN in comparison to those from healthy donors. Clinically achievable concentrations of ibrutinib $(0.5 \mu \mathrm{M})^{7}$ significantly reduced SDF1 $\alpha$-stimulated migration in JAK2-V617F MPN granulocytes (Figure $3 \mathrm{~A}$, right panel). Of note, in comparison to granulocytes from healthy donors, granulocytes isolated from CALR-mutated MPN patients demonstrated no detectable changes with respect to basal or SDF1 $\alpha$-induced transmigration (Figure $3 \mathrm{~B}$ ).

Transmigration of leukocytes and of progenitor cells is crucial to the process of extramedullary hematopoiesis. This process is regulated by chemokines such as SDF1 $\alpha$ and is mediated by the integrins LFA1 and VLA4. Recently, we showed that JAK2-V617F but not mutated CALR stimulates integrin signaling via activation of the small GTPase Rap1, resulting in increased binding of granulocytes to ICAM-1 and VCAM-1 (abundantly expressed in spleen). ${ }^{20}$ Thus, it is tempting to speculate that differences in chemotaxis in concert with differential integrin binding of JAK2-V617F versus CALR mutated leukocytes might contribute to extramedullary hematopoiesis. This hypothesis is in line with recent data showing that in primary myelofibrosis, the risk of splenomegaly is less pronounced in CALR-mutated patients than in JAK2-V617F-positive individuals. ${ }^{21}$

In conclusion, our data demonstrate that JAK2-V617F kinase, via its signaling intermediates BTK, PI3K/AKT, PLC $\gamma 1$, and RhoA, collaborates with chemokine SDF1 $\alpha$ and regulates cell migration. These findings expand our current understanding of the physiological role of activated JAK2-V617F signaling. The data further provide a rationale for investigating the contribution of these downstream molecules in abnormal cell motility of JAK2V617F-positive myeloid progenitors and stem cells migrating from bone marrow to peripheral blood and to extramedullary organs. The findings may also be useful to the clinical exploration of ruxolitinib-ibrutinib combinations to inhibit abnormal migration and homing of the JAK2-V617F-positive clone in MPN. Future studies are warranted to clarify the molecular mechanisms and clinical potential of these targets.

Subbaiah Chary Nimmagadda, ${ }^{1,2,3}$ Stephanie Frey, ${ }^{1,2}$ Peter Müller, ${ }^{1,2}$ Denise Wolleschak, ${ }^{1,2}$ Sönke Weinert, ${ }^{4}$ Ulrich Keller, ${ }^{3,5,6}$ Bärbel Edelmann $n^{1,2}$ and Thomas Fischer ${ }^{1,2}$

'Department of Hematology and Oncology, Medical Center, Otto-von-Guericke University, Magdeburg; ${ }^{2} H e a l t h$ Campus Immunology, Infectiology and Inflammation, Otto-von-GuerickeUniversity, Magdeburg; ${ }^{3}$ Medical Clinic and Polyclinic III, Klinikum rechts der Isar, Technical University Munich; ${ }^{4}$ Department of Cardiology and Angiology, Medical Center, Otto-von-Guericke University, Magdeburg; 'Department of Hematology, Oncology and Tumor Immunology, Campus Benjamin Franklin, Charité

Universitätsmedizin, Berlin and ${ }^{6}$ German Cancer Research Center (DKFZ) and German Cancer Consortium (DKTK), Heidelberg, Germany

Funding: this project was funded by grants from DFG (SFB854, project $A 20$ to TF and SFB1335, project $P 3$ to $U K)$ and $B M B F$ (e:Bio JAK-Sys to TF).

Acknowledgments: the authors would like to thank: Prof. Kristian Bowles and Dr. Lyubov Zaitseva (Norwich Medical School, UK) for their generous gift of P GL4-empty and PGL4-BTK-promoter constructs and Corinna Fahldieck, Uta Schönborn and Anja Sammt for their excellent technical support.

Correspondence: THOMAS FISCHER.

thomas.fischer@med.ovgu.de

doi:10.3324/haematol.2018.201921 
Information on authorship, contributions, and financial \& other disclosures was provided by the authors and is available with the online version of this article at www. haematologica.org.

\section{References}

1. Cross NCP. Genetic and epigenetic complexity in myeloproliferative neoplasms. Hematology Am Soc Hematol Educ Program. 2011;2011:208-214.

2. Hermouet S, Bigot-Corbel E, Gardie B. Pathogenesis of myeloproliferative neoplasms: role and mechanisms of chronic Inflammation. Mediators Inflamm. 2015;145293.

3. Verstovsek S, Kantarjian H, Mesa RA, et al. Safety and efficacy of INCB018424, a JAK1 and JAK2 inhibitor, in myelofibrosis. New Engl J Med. 2010;363(12):1117-1127.

4. Khan M, Siddiqi R, Gangat N. Therapeutic options for leukemic transformation in patients with myeloproliferative neoplasms. Leuk Res. 2017;63:78-84.

5. Schnoder TM, Eberhardt J, Koehler M, et al. Cell autonomous expression of CXCL-10 in JAK2V617F-mutated MPN. J Cancer Res Clin. 2017; 143(5):807-820.

6. Murray MY, Zaitseva L, Auger MJ, et al. Ibrutinib inhibits BTK-driven NF-kappa B p65 activity to overcome bortezomib-resistance in multiple myeloma. Cell Cycle. 2015;14(14):2367-2375.

7. Nimmagadda SC, Frey S, Edelmann B, et al. Bruton's tyrosine kinase and RAC1 promote cell survival in MLL-rearranged acute myeloid leukemia. Leukemia. 2018;32(3):846-849.

8. Schmidt U, van den Akker E, Parren-van Amelsvoort M, et al. Btk is required for an efficient response to erythropoietin and for SCF-controlled protection against TRAIL in erythroid progenitors. J Exp Med. 2004;199(6):785-795.

9. Schnoder TM, Arreba-Tutusaus P, Griehl I, et al. Epo-induced erythroid maturation is dependent on Plc gamma 1 signaling. Cell Death Differ. 2015;22(6):974-985

10. Yu L, Mohamed AJ, Simonson OE, et al. Proteasome-dependent autoregulation of Bruton tyrosine kinase (Btk) promoter via NFkappaB. Blood. 2008;111(9):4617-4626.

11. de Gorter DJJ, Beuling EA, Kersseboom R, et al. Bruton's tyrosine kinase and phospholipase C gamma 2 mediate chemokine-controlled B cell migration and homing. Immunity. 2007;26(1):93-104.

12. Zaitseva L, Murray MY, Shafat MS, et al. Ibrutinib inhibits SDF1/CXCR4 mediated migration in AML. Oncotarget. 2014, 5(20):9930-9938.

13. Abdelouahab H, Zhang YY, Wittner M, et al. CXCL12/CXCR4 pathway is activated by oncogenic JAK2 in a PI3K-dependent manner Oncotarget. 2017;8(33):54082-54095.

14. Wang XL, Cho SY, Hu CS, et al. C-X-C motif chemokine 12 influences the development of extramedullary hematopoiesis in the spleens of myelofibrosis patients. Exp Hematol. 2015;43(2):100-109.

15. Rushworth SA, Murray MY, Zaitseva L, et al. Identification of Bruton's tyrosine kinase as a therapeutic target in acute myeloid leukemia. Blood. 2014;123(8):1229-1238.

16. Luo IX, Li DY, Wei $\mathrm{D}$, et al. RhoA and RhoC are involved in stroma cell-derived factor-1-induced cell migration by regulating F-actin redistribution and assembly. Mol Cell Biochem. 2017;436(1-2):13-21.

17. Mao JH, Xie W, Yuan HD, et al. Tec/Bmx non-receptor tyrosine kinases are involved in regulation of Rho and serum response factor by G alpha 12/13. Embo J. 1998;17(19):5638-5646.

18. Socoro-Yuste N, Cokic VP, Mondet J, et al. Quantitative proteome heterogeneity in myeloproliferative neoplasm subtypes and association with JAK2 mutation status. Mol Cancer Res. 2017;15(7):852861.

19. Sit ST, Manser E. Rho GTPases and their role in organizing the actin cytoskeleton. J Cell Sci. 2011;124(Pt 5):679-683.

20. Edelmann B, Gupta N, Schnoeder TM, et al. JAK2-V617F promotes venous thrombosis through beta1/beta2 integrin activation. J Clin Invest. 2018;128(10):4359-4371.

21. Pei YO, Wu Y, Wang F, et al. Prognostic value of CALR vs. JAK2V617F mutations on splenomegaly, leukemic transformation, thrombosis, and overall survival in patients with primary fibrosis: a meta-analysis. Ann Hematol. 2016;95(9):1391-1398. 